categorised by pre-specified criteria, were used for the primary analysis.

Results 16 cross-sectional data-sets resulting in four sets of repeated cross-sectional studies were identified. Repeated studies conducted in Chennai, rural Tamil Nadu, Mauritius and Singapore ( $n=30$ 399), provided time-trend information. Three showed a statistically significant increase in diabetes prevalence $(p<0.001)$ while IGT prevalence fell significantly in two $(\mathrm{p}<0.05)$, and was stable in the remainder.

Conclusion This novel systematic review is the first to assess secular trends of pre-diabetes in any population. The data shows diabetes prevalence is rising in South Asians while IGT prevalence is either stable or falling. Recent environmental or lifestyle changes could favour an increased rate of conversion from IGT to diabetes or for individuals to progress directly from normal glycaemic status to diabetes. While the natural history of diabetes remains unclear, these findings have implications for health systems planning future treatment for diabetes and pre-diabetes.

\section{P2-132 INTERGENERATIONAL INFLUENCES ON DIABETES IN A DEVELOPING POPULATION: THE GUANGZHOU BIOBANK COHORT STUDY}

doi:10.1136/jech.2011.142976i.67

\begin{abstract}
${ }^{1} \mathrm{M}$ Schooling, ${ }^{*}{ }^{2} \mathrm{~S}$ Kavikondala, ${ }^{3} \mathrm{C}$ Jiang, ${ }^{3} \mathrm{~W}$ Zhang, ${ }^{4} \mathrm{~K}$ K Cheng, ${ }^{2} \mathrm{~T} \mathrm{H}$ Lam, ${ }^{2} \mathrm{G}$ Leung. ${ }^{1}$ University of Hong Kong, China; ${ }^{2}$ Department of Community Medicine and School of Public Health, Li Ka Shing Faculty of Medicine, The University of Hong Kong, Hong Kong SAR, China; ${ }^{3}$ Guangzhou Occupational Diseases Prevention and Treatment Centre, Guangzhou Number 12 Hospital, Guangzhou, Guangdong, China; ${ }^{4}$ Department of Public Health and Epidemiology, University of Birmingham, Birmingham, UK
\end{abstract}

Introduction Intergenerational "mismatch" has been suggested as being relevant to the emergent epidemic of diabetes in developing populations. Conversely, constrained growth conditions over generations may also increase susceptibility to diabetes. In a rapidly developing southern Chinese population, we tested whether maternal environment, proxied by maternal literacy, or family socioeconomic position (SEP), proxied by paternal literacy, were associated with fasting blood glucose and diabetes. To assess if intergenerational mismatch contributed, we tested whether the associations were modified by life course SEP.

Methods In 19818 older ( $\geq 50$ years) adults from the Guangzhou Biobank Cohort Study (phases 2 and 3) examined in 2005-2008, we used multivariable censored and logistic regression to assess the associations of maternal and paternal literacy with fasting blood glucose and diabetes and whether these associations varied by sex, age or life course SEP.

Results Adjusted associations of maternal, but not paternal, literacy was negatively associated with fasting blood glucose $(-0.07,95 \% \mathrm{CI}$ -0.13 to -0.02$)$ and diabetes $(0.92,95 \%$ CI 0.83 to 1.02$)$. These associations did not vary by sex, age or life course SEP.

Conclusions Better living conditions over generations may reduce the risk of diabetes, consistent with the high levels of diabetes in recently developed or developing populations.

\section{P2-133 MUSCLE MASS IS ASSOCIATED WITH DIABETES IN A DEVELOPING POPULATION: THE GUANGZHOU BIOBANK COHORT STUDY}

doi:10.1136/jech.2011.142976i.68

\footnotetext{
${ }^{1} \mathrm{M}$ Schooling, ${ }^{2} \mathrm{~S}$ Kavikondala, ${ }^{3} \mathrm{C}$ Jiang, ${ }^{3} \mathrm{~W}$ Zhang, ${ }^{4} \mathrm{~K}$ K Cheng, ${ }^{2} \mathrm{~T} \mathrm{H}$ Lam, ${ }^{2} \mathrm{G}$ Leung, ${ }^{2} \mathrm{~S}$ Mary. ${ }^{1}$ University of Hong Kong, China; ${ }^{2}$ Department of Community Medicine and School of Public Health, Li Ka Shing Faculty of Medicine, The University of Hong Kong, Hong Kong SAR, China; ${ }^{3}$ Guangzhou Occupational Diseases Prevention and Treatment
}

Centre, Guangzhou Number 12 Hospital, Guangzhou, Guangdong, China; ${ }^{4}$ Department of Public Health and Epidemiology, University of Birmingham, Birmingham, UK

Introduction Asian populations tend to be more susceptible to type 2 diabetes. In Asians low muscle-mass may also be a contributory factor. In a rapidly developing southern Chinese population, we examined whether skeletal muscle-mass, proxied by serum creatinine, was associated with diabetes or adiposity.

Methods In 9768 older ( $\geq 50$ years) adults from the Guangzhou Biobank Cohort Study (phase 1) examined in 2003-2004, we used multivariable logistic, linear and censored regression to assess the adjusted associations of serum creatinine with diabetes, waist-hip ratio, body mass index and fasting blood glucose. We also examined whether these associations varied by sex and age.

Results The association of serum creatinine with diabetes did not vary by sex. Serum creatinine was negatively associated with diabetes (OR 0.65 , 95\% CI 0.56 to 0.75 ) and fasting glucose $(-0.43 \mathrm{mmol} / \mathrm{l}, 95 \% \mathrm{CI}-0.53$ to -0.34$)$ for highest compared with lowest tertile, adjusted for age, education, occupation and lifestyle. Serum creatinine had sex-specific associations with waist-hip ratio, negatively associated $(-0.10 \mathrm{SDs}, 95 \% \mathrm{CI}-0.15$ to -0.04$)$ in women only.

Conclusions The observed pattern of associations is compatible with the hypothesis that nutritionally driven increases in muscle-mass, reduces diabetes risk while having sex-specific effects on central obesity, with corresponding implications for prevention.

\section{P2-134 EFFECTIVENESS OF WORK-SITE BEHAVIOURAL INTERVENTION PROGRAM FOR EMPLOYEES WITH METABOLIC SYNDROME AND ITS HIGH RISK PROFILE}

doi:10.1136/jech.2011.142976i.69

K Kayaba, ${ }^{*}$ Y Kikuchi, Y Miura. Saitama Prefectural University, Koshigaya, Japan

Introduction Japanese government introduced behavioural approach for modifying unfavourable life styles leading to metabolic syndrome (MetS) into community health promotion in 2008. Previous studies have reported its efficacy in community settings. However, few studies examined effectiveness of work-site behavioural intervention for employees.

Methods A total of 311 male workers of a health insurance company were recruited. They were classified as high risk group of Mets by measuring BMI, blood pressure, $\mathrm{HbA1c}, \mathrm{HDL}$ and $\mathrm{LDL}$ cholesterol levels at the annual health examination in 2008. A subgroup of them were recommended to participate in a health promotion activity with behavioural approach aiming diet and physical activity modification (intervention group; 86 persons). After 1-year follow-up, prevalence of MetS and levels of the previous factors were compared between the intervention group and non-intervention group (225 persons).

Results After adjusting for age, no statistically significant difference of the prevalence of MetS and levels of the previous factors was found between the group at the baseline examination. In the 2009 examination, BMI, systolic and diastolic blood pressure and LDL cholesterol levels decreased significantly in the intervention group while HbA1c level increased and HDL cholesterol level decreased in the non-intervention group. Prevalence of Mets decreased in the intervention group ( $-23.3 \%$ : $95 \% \mathrm{CI}-34.2$ to $-12.2 \%$ ) and also did in the non-intervention group ( $-16.8 \%$ : -23.5 to $-10.3 \%)$. Difference in change of the prevalence in each group (6.5\%: -3.7 to $16.7 \%)$ was in borderline significance.

Conclusion This quasi-experimental study at worksite suggested effectiveness of work-site behavioural intervention for employees with MetS in Japan. 